\title{
A Concise Enantioselective Synthesis of the Aminocyclictol Core of Hygromycin A
}

Timothy J. Donohoe,* Richard J. Pye, Peter D. Johnson and Martine Keenan

\section{Supplementary Material}

Key procedures are included:

\section{Mitsunobu Reaction (8)}

To a stirred solution of alcohol (7) $(7.50 \mathrm{~g}, 34.1 \mathrm{mmol})$ in THF (250 mL) under an atmosphere of nitrogen was added sequentially tributyl phosphine (15.1 mL, 61.4 mmol) and para-nitrobenzoic acid $(6.83 \mathrm{~g}, 40.9 \mathrm{mmol})$. Once dissolved, di-tert-butylazodicarboxylate (14.1 $\mathrm{g}, 61.4 \mathrm{mmol}$ ) was added in one portion and the resulting solution stirred at room temperature for 45 mins. The solution was diluted with water $(150 \mathrm{~mL})$ and extracted with ether $(100 \mathrm{~mL} \times 2,50 \mathrm{~mL}$ $\mathrm{x} 2)$. The combined organics were washed with brine $(50 \mathrm{~mL})$, dried over $\mathrm{MgSO}_{4}$ and concentrated in vacuo to furnish an orange syrup. To this was added $\mathrm{MeOH}$ until precipitation occurred. The mixture was allowed to stand at RT for $2 \mathrm{~h}$ then placed in a freezer for $15 \mathrm{~h}$. The solid was filtered, washed with ice-cold $\mathrm{MeOH}$ and dried under vacuum to furnish the title compound as a pale green solid (9.85 $\mathrm{g}, 78 \%$ ). Further product was isolated by concentrating the filtrate onto silica and purifying by flash column chromatography, eluting with petrol-EtOAC (9:1), to yield $465 \mathrm{mg}$ of the title compound. The combined yield was $10.32 \mathrm{~g}, 83 \%$. m.p. $88-89^{\circ} \mathrm{C} ;[\alpha]_{\mathrm{D}}^{20}-227.8\left(\mathrm{C}=1.33, \mathrm{CHCl}_{3}\right) ;$ IR $v_{\max }(\mathrm{KBr})$ 1723，1528，1270，1242; ${ }^{1} \mathrm{H}$ NMR $\delta_{\mathrm{H}}\left(400 \mathrm{MHz} ; \mathrm{CDCl}_{3}\right)$ 8.31-8.19 $(4 \mathrm{H}, \mathrm{m}, \mathrm{Ar}), 6.11\left(1 \mathrm{H}, \mathrm{dd}, J 5.5\right.$ and $3.0, \mathrm{C}^{2} \mathrm{H}=\mathrm{C}^{3} \underline{\mathrm{H}}$ ) , 5.95 ( $1 \mathrm{H}$, $\mathrm{dd}, J 5.5$ and 3.0, $\left.\mathrm{C}^{2} \mathrm{H}=\mathrm{C}^{3} \mathrm{H}\right), 5.90$ ( $1 \mathrm{H}, \mathrm{ddd}, J 10.5,4.5$ and 1.5, $\left.\mathrm{C}^{6} \mathrm{H}=\mathrm{C}^{7} \underline{\mathrm{H}}\right), \quad 5.84 \quad\left(1 \mathrm{H}, \quad \mathrm{dd}, \quad J 10.5\right.$ and $\left.3.0, \mathrm{C}^{6} \mathrm{H}=\mathrm{C}^{7} \mathrm{H}\right), \quad 5.62$ $(1 \mathrm{H}, \mathrm{ddd}, J 8.5,3.5$ and $2.0, \mathrm{CHOBz}), 5.33(1 \mathrm{H}, t, J 4.5$, 
CHOAC), $3.10-3.05\left(2 \mathrm{H}, \mathrm{m}, \mathrm{C}^{1} \mathrm{H}\right.$ and $\left.\mathrm{C}^{4 a} \mathrm{H}\right), 2.87$ ( $\left.1 \mathrm{H}, \mathrm{br} \mathrm{s}, \mathrm{C}^{4} \mathrm{H}\right)$, $2.81\left(1 \mathrm{H}, \mathrm{dt}, \mathrm{J} 10.0\right.$ and $\left.4.0, \mathrm{C}^{8 \mathrm{a}} \mathrm{H}\right), 2.13(3 \mathrm{H}, \mathrm{s}, \mathrm{Me}), 1.44$ $\left(1 \mathrm{H}, \mathrm{dt}, \mathrm{J} 8.5\right.$ and $\left.2.0, \mathrm{CH}_{\mathrm{A}} \mathrm{H}_{\mathrm{B}}\right), 1.38\left(1 \mathrm{H}\right.$, br $\left.\mathrm{m}, \mathrm{CH}_{\mathrm{A}} H_{\mathrm{B}}\right) ;{ }^{13} \mathrm{C} \mathrm{NMR}$ $\delta_{\mathrm{C}}\left(\mathrm{CDCl}_{3}\right) 170.9,164.6,151.0,137.3,136.2,134.2,132.7$, 131.1, 129.3, 123.9, 71.9, 68.6, 49.8, 47.2, 46.2, 43.9, $41.7,21.6 ; \mathrm{m} / \mathrm{z}$ (CI) $387\left(\mathrm{M}+\mathrm{NH}_{4}^{+}\right), 220(\mathrm{M}-\mathrm{COAr}), 203$ (MOCOAr) and 143 (100\%, M-OCOAr -OAC); HRMS for $\mathrm{C}_{20} \mathrm{H}_{23} \mathrm{~N}_{2} \mathrm{O}_{6}$ requires 387.1556 , found 387.1562 .

\section{Bromo ether (11)}

To a stirred solution of diol (9) (4.79 $\mathrm{g}, 26.9 \mathrm{mmol})$ in dichloromethane $(250 \mathrm{~mL})$ at $0^{\circ} \mathrm{C}$ was added recrystallised $\mathrm{N}$ bromosuccinimide $(5.27 \mathrm{~g}, 29.6 \mathrm{mmol})$ and the resulting solution was stirred for $45 \mathrm{~min}$. The crude material was dry loaded onto silica and purified by flash column chromatography, eluting with petrol:ethyl acetate (1:1), to furnish the title compound as a colourless oil (6.429, 93\%); $R_{\mathrm{f}}(1: 1$ EtOAC - petrol $) 0.30 ;[\alpha]_{\mathrm{D}} 119\left(\mathrm{c}=1.14, \mathrm{CHCl}_{3}\right)$; IR $v_{\max }\left(\mathrm{CHCl}_{3}\right) 3393(\mathrm{OH}), 2968$ and $2888(\mathrm{CH}) ;{ }^{1} \mathrm{H}$ NMR $\delta_{\mathrm{H}}(400 \mathrm{MHz}$; $\left.\mathrm{CDCl}_{3}\right) 6.04\left(1 \mathrm{H}, \mathrm{ddd}, \mathrm{J} 10.0,5.5\right.$ and $\left.1.5, \mathrm{C}^{6} \mathrm{H}=\mathrm{C}^{7} \mathrm{H}\right), 5.95$ ( $1 \mathrm{H}, \mathrm{ddd}, \mathrm{J} 10.0,3.0$ and $\left.1.0, \mathrm{C}^{6} \mathrm{H}=\mathrm{C}^{7} \mathrm{H}\right), 4.51$ ( $1 \mathrm{H}, \mathrm{d}, \mathrm{J}$ 5.0, $\left.\mathrm{C}^{3} \mathrm{HOR}\right), 4.36\left(1 \mathrm{H}, \mathrm{t}, \mathrm{J} 5.0, \mathrm{C}^{5} \mathrm{H}\right), 4.10\left(1 \mathrm{H}, \mathrm{m}, \mathrm{C}^{8} \mathrm{H}\right)$, $3.61\left(1 \mathrm{H}, \mathrm{d}, J 2.5, \mathrm{C}^{2} \mathrm{HBr}\right), 2.88(1 \mathrm{H}, \mathrm{tq}, J 5.0$ and 1.5 , $\left.\mathrm{C}^{4} \mathrm{H}\right), 2.55-2.50\left(2 \mathrm{H}, \mathrm{m}, \mathrm{C}^{1} \mathrm{H}\right.$ and $\left.\mathrm{OH}\right), 2.40(1 \mathrm{H}, \mathrm{dt}, \mathrm{J} 10.5$ and 5.0, $\left.\mathrm{C}^{4 a} \mathrm{H}\right), 2.31\left(1 \mathrm{H}\right.$, ddd, $J 11.0,3.5$ and $\left.1.5, \mathrm{C}^{8 \mathrm{a}} \mathrm{H}\right)$, $2.08\left(1 \mathrm{H}, d t, J 11.0\right.$ and $\left.1.5, C H_{A} H_{B}\right)$ and $1.65(1 \mathrm{H}, \mathrm{dm}$, $\left.\mathrm{CH}_{\mathrm{A}} \mathrm{H}_{\mathrm{B}}\right) ;{ }^{13} \mathrm{C} \quad \mathrm{NMR} \quad \delta_{\mathrm{C}}\left(\mathrm{CDCl}_{3}\right) \quad 132.4,128.1,89.4 \quad$ ( $\left.\mathrm{C}^{3} \mathrm{OR}\right), 71.9$ $\left(\mathrm{C}^{5} \mathrm{OR}\right), 64.2\left(\mathrm{C}^{8} \mathrm{OH}\right), 55.0\left(\mathrm{C}^{2} \mathrm{Br}\right), 49.1\left(\mathrm{C}^{4}\right), 48.0\left(\mathrm{C}^{1}\right), 45.7$ $\left(\mathrm{C}^{8 \mathrm{a}}\right), 34.2\left(\mathrm{C}^{4 a}\right)$ and $34.1\left(\mathrm{CH}_{2}\right) ; \mathrm{MS} \mathrm{m} / \mathrm{z}(\mathrm{CI}) 258,256\left(\mathrm{M}^{+}\right)$, 194, $177\left(100 \%, \mathrm{M}^{+}-\mathrm{Br}\right)$ and $161\left(\mathrm{MH}^{+}-\mathrm{Br}-\mathrm{OH}\right)$; HRMS (CI) Found: $\mathrm{M}^{+}, 256.0107 . \mathrm{C}_{11} \mathrm{H}_{13} \mathrm{O}_{2} \mathrm{Br}^{+}$requires $M, 256.0099$. 


\section{Carbamate (12)}

To a stirred solution of alcohol (11) (6.42 $\mathrm{g}, 24.98 \mathrm{mmol})$ in dichloromethane at $0^{\circ} \mathrm{C}$ was added dropwise trichloroacetyl isocyanate $(3.56 \mathrm{~mL}, 29.98 \mathrm{mmol})$. The resulting solution was stirred for $1.75 \mathrm{~h}$ and then concentrated in vacuo. The residue was diluted with $\mathrm{MeOH}(60 \mathrm{~mL})$, cooled to $0^{\circ} \mathrm{C}$ and a solution of potassium carbonate $(10.34 \mathrm{~g}, 74.94 \mathrm{mmol})$ in $\mathrm{H}_{2} \mathrm{O}$ (15 mL) was added. The resulting suspension was stirred at $0^{\circ} \mathrm{C}$ for $2 \mathrm{~h}$, then at $\mathrm{RT}$ for $16 \mathrm{~h}$. The reaction was concentrated in vacuo, diluted with $\mathrm{H}_{2} \mathrm{O}$ (50 $\left.\mathrm{mL}\right)$ and brine (50 $\mathrm{mL}$ ) and extracted with dichloromethane (50 mL x5). The combined organic extracts were dried over $\mathrm{MgSO}_{4}$ and concentrated in vacuo. The crude material was purified by recrystallisation from EtOH to furnish the title compound as a white solid $(6.81 \mathrm{~g}, 91 \%)$;

m.p. $124-126{ }^{\circ} \mathrm{C} ; \quad[\alpha]_{\mathrm{D}} 106\left(\mathrm{C}=1.05, \mathrm{CHCl}_{3}\right) ;$ IR $v_{\max }\left(\mathrm{CHCl}_{3}\right)$ $3459,3344\left(\mathrm{br}, \mathrm{NH}_{2}\right), 2972(\mathrm{CH})$ and $1716(\mathrm{C}=\mathrm{O}) ;{ }^{1} \mathrm{H} \mathbf{N M R} \delta_{\mathrm{H}}(400$ $\left.\mathrm{MHz} ; \mathrm{CDCl}_{3}\right) 6.16\left(1 \mathrm{H}\right.$, ddd, J $10.0,6.0$ and $\left.2.0, \mathrm{C}^{6} \mathrm{H}=\mathrm{C}^{7} \mathrm{H}\right)$, $5.90\left(1 \mathrm{H}, \mathrm{dd}, \mathrm{J} 10.0\right.$ and $\left.3.5, \mathrm{C}^{6} \mathrm{H}=\mathrm{C}^{7} \mathrm{H}\right), 5.07$ ( $1 \mathrm{H}$, dt, J 3.0 and $\left.1.5, \mathrm{C}^{8} \mathrm{HOCO}\right), 4.92\left(2 \mathrm{H}\right.$, br s, $\left.\mathrm{NH}_{2}\right), 4.53(1 \mathrm{H}, \mathrm{d}, \mathrm{J}$ 4.5, $\left.\mathrm{C}^{3} \mathrm{HOR}\right), 4.40$ ( $\left.1 \mathrm{H}, \mathrm{t}, \mathrm{J} 6.0, \mathrm{C}^{5} \mathrm{HOR}\right), 3.64(1 \mathrm{H}, \mathrm{d}, \mathrm{J}$ 2.5, $\left.\mathrm{C}^{2} \mathrm{HBr}\right), 2.89\left(1 \mathrm{H}, \mathrm{td}, \mathrm{J} 5.0\right.$ and $\left.1.0, \mathrm{C}^{4} \mathrm{H}\right), 2.69$ ( $1 \mathrm{H}$, $\left.\mathrm{d}, \mathrm{J} 1.0, \mathrm{C}^{1} \mathrm{H}\right), 2.39\left(\mathrm{H}, \mathrm{dt}, \mathrm{J} 10.5\right.$ and $\left.5.0, \mathrm{C}^{4 \mathrm{a}} \mathrm{H}\right), 2.30$ ( $1 \mathrm{H}, \mathrm{dd}, J 11.0$ and $\left.3.5, \mathrm{C}^{8 \mathrm{a}} \mathrm{H}\right), 2.09\left(1 \mathrm{H}, \mathrm{d}, J 11.0, \mathrm{CH}_{\mathrm{A}} \mathrm{H}_{\mathrm{B}}\right)$ and $1.64\left(1 \mathrm{H}, \mathrm{ddd}, J 11.0,1.5\right.$ and $\left.1.0, \mathrm{CH}_{\mathrm{A}} H_{\mathrm{B}}\right) ;{ }^{13} \mathrm{C}$ NMR $\delta_{\mathrm{C}}\left(\mathrm{CDCl}_{3}\right) ; 156.3(\mathrm{C}=\mathrm{O}), 130.0 \quad\left(\mathrm{C}^{6} \mathrm{H}=\mathrm{C}^{7} \mathrm{H}\right), 128.5 \quad\left(\mathrm{C}^{6} \mathrm{H}=\mathrm{C}^{7} \mathrm{H}\right), \quad 89.4$ $\left(\mathrm{C}^{3} \mathrm{OR}\right), 71.8$ ( $\left.\mathrm{C}^{5} \mathrm{OR}\right), 67.3\left(\mathrm{C}^{8} \mathrm{OR}\right), 54.7\left(\mathrm{C}^{2} \mathrm{Br}\right), 49.2\left(\mathrm{C}^{4} \mathrm{H}\right)$, $47.9\left(\mathrm{C}^{1} \mathrm{H}\right), 43.2\left(\mathrm{C}^{8 \mathrm{a}} \mathrm{H}\right), 34.1\left(\mathrm{C}^{4 a} \mathrm{H}\right)$ and $34.0\left(\mathrm{CH}_{2}\right) ; \mathbf{M S} \mathrm{m} / \mathrm{z}$ (CI) 319, $317\left(\mathrm{MNH}_{4}^{+}\right), 302,301\left(\mathrm{MH}^{+}\right), 258,256\left(100 \%, \mathrm{M}^{+}-\right.$ $\left.\mathrm{CONH}_{2}\right), 241$ and $239\left(\mathrm{M}^{+}-\mathrm{OCONH}_{2}\right)$; HRMS (CI) Found: $\mathrm{MH}^{+}$, $300.0236 . \mathrm{C}_{12} \mathrm{H}_{15} \mathrm{NO}_{3} \mathrm{Br}^{+}$requires $M, 300.0235$. 


\section{Oxazolidinone (13)}

Carbamate (12) was dissolved in propan-1-ol (120 mL). A solution of sodium hydroxide $\left(733 \mathrm{mg}\right.$, $18.34 \mathrm{mmol}$ ) in $\mathrm{H}_{2} \mathrm{O}$ (80 $\mathrm{mL}$ ) was made and $78 \mathrm{~mL}$ of this solution was added to the above carbamate solution. The resulting mixture was stirred for $5 \mathrm{~min}$, then t-butyl hypochlorite $(2.28 \mathrm{~mL}, 19.93 \mathrm{mmol})$ was added and the resulting solution stirred for $5 \mathrm{~min}$. To this was added sodium citrate (1.17 g, $20 \mathrm{~mol} \%$ ) and the solution stirred for 5 min. Finally a solution of potassium osmate (293 mg, $4 \mathrm{~mol} \%$ ) in the remainder of the sodium hydroxide solution ( $2 \mathrm{~mL}$ ) was added. The resulting solution was stirred at $\mathrm{RT}$ for $20 \mathrm{~h}$. To the reaction was added sodium sulfite $(10 \mathrm{~g})$ and the mixture was stirred for 30 min. The mixture was diluted with brine and extracted with EtOAC $(x 5)$. The combined organics were washed with brine, dried over sodium sulfate and concentrated in vacuo. The crude material was purified by flash column chromatography on silica, eluting with petrol:EtOAc (1:1 then $0: 1)$ to furnish the title compound as white solid (4.24 9, 67\%). Starting material (930 $\mathrm{mg}, 16 \%$ ) was also recovered;

$R_{\mathrm{f}} \quad(\mathrm{EtOAC}) \quad 0.14 ; \mathrm{m} \cdot \mathrm{p} .220{ }^{\circ} \mathrm{C} \quad($ dec. $) ; \quad[\alpha]_{\mathrm{D}} 85 \quad(\mathrm{c}=1.47$ ， pyridine); IR $v_{\max }\left(\mathrm{CHCl}_{3}\right) 3357$ (br, OH and $\mathrm{NH}$ ), 2972, 2886 $(\mathrm{CH})$ and $1742(\mathrm{C}=\mathrm{O}) ;{ }^{1} \mathrm{H}$ NMR $\delta_{\mathrm{H}}\left(400 \mathrm{MHz} ; d^{5}\right.$-pyridine) 7.55 (1 $\mathrm{H}, \mathrm{d}, \mathrm{J} 4.0, \mathrm{NH}), 4.80\left(1 \mathrm{H}, \mathrm{br} \mathrm{m}, \mathrm{C}^{5} \mathrm{HOR}\right), 4.65$ ( $1 \mathrm{H}, \mathrm{br} \mathrm{d}$, J 3.5, $\left.\mathrm{C}^{3} \mathrm{HOR}\right), 4.50$ ( $1 \mathrm{H}$, br m, $\left.\mathrm{C}^{8} \mathrm{HOR}\right), 4.38$ ( $1 \mathrm{H}$, br m, $\left.\mathrm{C}^{7} \mathrm{HNH}\right), \quad 4.06\left(1 \mathrm{H}, \mathrm{m}, \mathrm{C}^{6} \mathrm{HOH}\right), 4.02\left(1 \mathrm{H}, \mathrm{s}, \mathrm{C}^{2} \mathrm{HBr}\right), 2.83$ (1 $\left.\mathrm{H}, \mathrm{m}, \mathrm{C}^{1} \mathrm{H}\right), 2.76-2.73\left(2 \mathrm{H}, \mathrm{m}, \mathrm{C}^{4} \mathrm{H}\right.$ and $\left.\mathrm{C}^{8 \mathrm{a}} \mathrm{H}\right), 2.44(1 \mathrm{H}, \mathrm{s}$, $\left.\mathrm{C}^{4 \mathrm{a}} \mathrm{H}\right), 1.98\left(1 \mathrm{H}, \mathrm{d}, J 11.0, \mathrm{CH}_{\mathrm{A}} \mathrm{H}_{\mathrm{B}}\right)$ and $1.52(1 \mathrm{H}, \mathrm{d}, J 10.5$, $\left.\mathrm{CH}_{\mathrm{A}} H_{\mathrm{B}}\right) ;{ }^{13} \mathrm{C}$ NMR $\delta_{\mathrm{C}}\left(d^{5}\right.$-pyridine) $161.3(\mathrm{C}=\mathrm{O}), 89.4 \quad\left(\mathrm{C}^{3} \mathrm{OR}\right), 80.5$ $\left(\mathrm{C}^{8} \mathrm{OR}\right), 76.5\left(\mathrm{C}^{5} \mathrm{OR}\right), 66.3\left(\mathrm{C}^{7} \mathrm{NH}\right), 57.0\left(\mathrm{C}^{2} \mathrm{Br}\right), 52.7\left(\mathrm{C}^{6} \mathrm{OH}\right)$, $48.4\left(\mathrm{C}^{8 \mathrm{a}} \mathrm{H}\right), \quad 48.1\left(\mathrm{C}^{4 a} \mathrm{H}\right), \quad 39.0\left(\mathrm{C}^{1} \mathrm{H}\right), \quad 35.2\left(\mathrm{C}^{4} \mathrm{H}\right)$ and 34.4 $\left(\mathrm{CH}_{2}\right)$; MS $\mathrm{m} / \mathrm{z}$ (ES) 315, $313\left(100 \%, \mathrm{M}-\mathrm{H}^{+}\right)$; HRMS (ES) Found: $\mathrm{MH}^{+}, 316.0191 . \mathrm{C}_{12} \mathrm{H}_{15} \mathrm{NO}_{4}{ }^{79} \mathrm{Br}^{+}$requires $M, 316.0184$. 


\section{Benzyl protected TA (14)}

To a stirred solution of (13) (4.20 g, $13.29 \mathrm{mmol})$ in DMF (35 mL) was added benzyl bromide (4.80 mL) and TBAI (50 mg). The solution was cooled to $0^{\circ} \mathrm{C}$ and sodium hydride $(1.60 \mathrm{~g}$, $66.66 \mathrm{mmol}$ ) was added in two equal portions over $10 \mathrm{~min}$. The reaction was warmed to $\mathrm{RT}$ after $30 \mathrm{~min}$ and stirred for a further $4 \mathrm{~h}$. The reaction was carefully quenched by addition of $\mathrm{H}_{2} \mathrm{O}$. The residue was washed with $\mathrm{H}_{2} \mathrm{O}$ and extracted with EtOAC $(\mathrm{x} 4)$. The combined organics were washed with brine, dried over $\mathrm{MgSO}_{4}$ and concentrated in vacuo. The crude material was purified by flash column chromatography on silica, eluting with petrol:EtoAc (98:2 then 4:1) to furnish the title compound as a white solid (4.98 $\mathrm{g}, 92 \%)$.

m.p. $\quad 168-171^{\circ} \mathrm{C} ; \quad[\alpha]_{\mathrm{D}}{ }^{20}-120.5\left(\mathrm{C}=0.64, \mathrm{CHCl}_{3}\right) ;$ IR $\boldsymbol{v}_{\max }(\mathrm{KBr})$ $1728 ;{ }^{1} \mathrm{H}$ NMR $\delta_{\mathrm{H}}\left(400 \mathrm{MHz} ; \mathrm{CDCl}_{3}\right) 7.42-7.19(10 \mathrm{H}, \mathrm{m}, \mathrm{Ar}), 4.77$ $\left(1 \mathrm{H}, \mathrm{d}, \mathrm{J} 15.6, \mathrm{CH}_{2} \mathrm{Bn}\right), 4.63\left(1 \mathrm{H}, \mathrm{d}, \mathrm{J} 12.0, \mathrm{CH}_{2} \mathrm{Bn}\right), 4.50-$ $4.46(3 \mathrm{H}, \mathrm{m}), 4.37(1 \mathrm{H}, t, J 4.0), 3.89(1 \mathrm{H}, t, J 4.0)$, $3.58-3.55(1 \mathrm{H}, \mathrm{m}), 3.53\left(1 \mathrm{H}, \mathrm{d}, \mathrm{J} 15.6, \mathrm{CH}_{2} \mathrm{Bn}\right), 3.42(1 \mathrm{H}$, $\mathrm{m}), 2.89(1 \mathrm{H}, \mathrm{m}), 2.75(1 \mathrm{H}, \mathrm{dd}, J 10.4,4.0), 2.58-2.51(2 \mathrm{H}$, $\mathrm{m}), 2.12(1 \mathrm{H}, \mathrm{d}, \mathrm{J} 11.2), 1.74(1 \mathrm{H}, \mathrm{d}, J 11.2), 1.60(1 \mathrm{H}$, $\mathrm{s}) ; \quad{ }^{13} \mathrm{C} \quad \mathrm{NMR} \quad \delta_{\mathrm{C}}\left(\mathrm{CDCl}_{3}\right) \quad 158.4,137.1,136.2,128.9,128.8$, $128.6,128.5,128.1,127.9,127.8,127.7,88.9,75.9,73.8$, $72.6,69.4,54.7,53.5,47.9,47.1,46.1,38.1,35.0,34.1$; $\mathrm{m} / z$ (CI) $556\left(100 \%, \mathrm{M}+\mathrm{MeCN}+\mathrm{NH}_{4}^{+}\right)$; HRMS for $\mathrm{C}_{26} \mathrm{H}_{27}{ }^{79} \mathrm{BrNO}_{4}$ requires 496.1123, found 496.1137.

\section{Bromo Ether Cleavage (15)}

To a solution of (14) (2.28 $\mathrm{g}, 46.06 \mathrm{mmol})$ in $\mathrm{MeOH}$ (70 $\mathrm{mL}$ ) was added zinc metal $(3.04 \mathrm{~g}, 46.77 \mathrm{mmol})$. To this was added 8 drops of glacial acetic acid and the resulting solution was refluxed for $4 \mathrm{~h}$, cooled to RT and filtered through a short pad of silica eluting with $\mathrm{MeOH}$. The 
organics were concentrated in vacuo and the crude material was purified by flash column chromatography on silica, eluting with petrol:EtOAC $(3: 2$ then $0: 1)$ to furnish the title compound as a colourless oil (1.65 g, 86\%).

$[\alpha]_{\mathrm{D}}^{20}-86.7\left(\mathrm{C}=1.03, \mathrm{CHCl}_{3}\right) ; \mathrm{IR} v_{\max }(\mathrm{KBr}) 3378,1717,1602$ ， $1577 ;{ }^{1} \mathrm{H} \operatorname{NMR} \delta_{\mathrm{H}}\left(400 \mathrm{MHz} ; \mathrm{CDCl}_{3}\right)$ 7.43-7.30 (5H, m, Ar), 7.29$7.22(3 \mathrm{H}, \mathrm{m}, \mathrm{Ar}), 7.13-7.11(2 \mathrm{H}, \mathrm{m}, \mathrm{Ar}), 6.31(1 \mathrm{H}, \mathrm{dd}, \mathrm{J}$ $5.6,3.2, \mathrm{CH}=\mathrm{CH}), 6.20(1 \mathrm{H}, \mathrm{dd}, J 5.5,3.2, \mathrm{CH}=\mathrm{CH}), 4.86$ $\left(1 \mathrm{H}, \mathrm{d}, \mathrm{J} 14.8, \mathrm{CH}_{2} \mathrm{Bn}\right), 4.58\left(1 \mathrm{H}, \mathrm{d}, \mathrm{J} 12.0, \mathrm{CH}_{2} \mathrm{Bn}\right), 4.48$ $(1 \mathrm{H}, \mathrm{m}), 4.42\left(1 \mathrm{H}, \mathrm{d}, \mathrm{J} 12.0, \mathrm{CH}_{2} \mathrm{Bn}\right), 4.22(1 \mathrm{H}, \mathrm{dd}, \mathrm{J} 9.6$, $6.8), 3.82-3.77(2 \mathrm{H}, \mathrm{m}), 3.73(1 \mathrm{H}, \mathrm{dd}, \mathrm{J} 9.6,4.4), 3.13$ $(1 \mathrm{H}, \mathrm{s}), 2.92(1 \mathrm{H}, \mathrm{s}) 2.79-2.76(2 \mathrm{H}, \mathrm{m}), 1.54-1.48(2 \mathrm{H}, \mathrm{m})$, $1.41(1 \mathrm{H}, \mathrm{d}, \mathrm{J} 8.0) ;{ }^{13} \mathrm{C} \operatorname{NMR} \delta_{\mathrm{C}}\left(\mathrm{CDCl}_{3}\right)$ 158.6, 153.1, 152.4， $151.8,137.6,137.2,136.5,135.9,128.7,128.1,127.8$, $127.7,75.2,74.4,72.0,67.0,53.5,51.4,46.6,45.7,45.2$, $43.7,43.4 ; \mathrm{m} / \mathrm{z}$ (CI) 476 (100\%, $\left.\mathrm{M}+\mathrm{MeCN}+\mathrm{NH}_{4}^{+}\right) ;$HRMS for $\mathrm{C}_{26} \mathrm{H}_{28} \mathrm{NO}_{4}$ requires 418.2018 , found 418.2013 .

\section{Retro Diels Alder (16)}

Compound (15) (728 mg, $1.75 \mathrm{mmol}$ ) was dissolved in diphenyl ether $(20 \mathrm{~mL})$ and refluxed gently for $1.5 \mathrm{~h}$. The reaction was cooled to RT and purified by flash column chromatography on silica, eluting with petrol-EtOAc (1:0 then 4:1) to furnish the title compound as an oil (543 mg, 88\%);

$[\alpha]_{D}^{20}+65.64\left(\mathrm{C}=0.66, \mathrm{CHCl}_{3}\right) ; \quad$ IR $v_{\max }(\mathrm{KBr}) 3382,1725$,

$1497 ;{ }^{1} \mathrm{H} N \mathrm{NMR} \delta_{\mathrm{H}}\left(400 \mathrm{MHz} ; \mathrm{CDCl}_{3}\right) 7.38-7.20$ (10H, m, Ar), 6.00 $(1 \mathrm{H}, \mathrm{m}, \mathrm{CH}=\mathrm{CH}), 5.72(1 \mathrm{H}, \mathrm{dt}, J 10.4,1.2, \mathrm{CH}=\mathrm{CH}), 4.91(1 \mathrm{H}$, $\mathrm{m}), 4.86\left(1 \mathrm{H}, \mathrm{d}, \mathrm{J} 15.2, \mathrm{C}_{2} \mathrm{Bn}\right), 4.53\left(1 \mathrm{H}, \mathrm{d}, J 12.0, \mathrm{CH}_{2} \mathrm{Bn}\right)$, $4.48\left(1 \mathrm{H}, \mathrm{d}, \mathrm{J} 12.0, \mathrm{CH}_{2} \mathrm{Bn}\right), 4.48-4.43(1 \mathrm{H}, \mathrm{m}), 4.32(1 \mathrm{H}, \mathrm{d}$, J 15.2, $\left.\mathrm{CH}_{2} \mathrm{Bn}\right), 4.21(1 \mathrm{H}, \mathrm{dd}, J 7.2,2.8), 3.48(1 \mathrm{H}, \mathrm{dd}, J$ 8.0, 2.8); ${ }^{13} \mathrm{C} \quad \mathrm{NMR} \delta_{\mathrm{C}}\left(\mathrm{CDCl}_{3}\right) 159.2,137.1,136.0,133.8$, $128.6,128.5,128.2,128.0,127.6,123.7,80.4,73.3,71.1$, 
$66.6,53.8,47.8 ; \mathrm{m} / z$ (CI) 410 (100\%, $\mathrm{M}+\mathrm{MeCN}+\mathrm{NH}_{4}^{+}$); HRMS for $\mathrm{C}_{21} \mathrm{H}_{22} \mathrm{NO}_{4}$ requires 352.1549, found 352.1553 .

\section{Osmate Ester $\mathrm{OH}$ (17)}

To a stirred solution of alkene (16) (184 $\mathrm{mg}, 0.53 \mathrm{mmol}$ ) in dichloromethane $(24 \mathrm{~mL})$ under an atmosphere of nitrogen was added $N, N, N, N$, tetramethylethyldiamine $(87.0 \mu \mathrm{L}, 0.58$ mmol) and the mixture was cooled to $-78^{\circ} \mathrm{C}$. To this was added dropwise a solution of $\mathrm{OsO}_{4}(140 \mathrm{mg}, 0.55 \mathrm{mmol})$ in dichloromethane ( $1 \mathrm{~mL}$ ) and the reaction was stirred for $2 \mathrm{~h}$. The solution was allowed to warm to RT, concentrated onto silica and the crude material purified by flash column chromatography, eluting with dichloromethane:MeOH (95:5), to furnish the title compound as a brown foam (379 mg, 100\%).

$\delta_{\mathrm{H}}\left(400 \mathrm{MHz}, \mathrm{CDCl}_{3}\right) 7.36-7.18(10 \mathrm{H}, \mathrm{m}, \mathrm{Ar}), 4.90(1 \mathrm{H}, \mathrm{dd}, J$ 8.8, 4.8, $\mathrm{CHOH}), 4.84\left(1 \mathrm{H}, \mathrm{d}, \mathrm{J} 15.2, \mathrm{CH}_{2} \mathrm{Ph}\right), 4.75(1 \mathrm{H}, \mathrm{d}, J$ $\left.11.6, \mathrm{CH}_{2} \mathrm{Ph}\right), 4.68(1 \mathrm{H}, \mathrm{t}, J 5.6, \mathrm{CHOOs}), 4.56(1 \mathrm{H}, \mathrm{dd}, J$ 11.2, 5.6, CHOC), $4.53(1 \mathrm{H}, \mathrm{m}, \mathrm{CHOOs}), 4.48(1 \mathrm{H}, \mathrm{d}, \mathrm{J} 12.0$, $\left.\mathrm{CH}_{2} \mathrm{Ph}\right), 4.01(1 \mathrm{H}$, app quart., $\mathrm{CHOBn}), 4.01(1 \mathrm{H}$, app. quart. , $\mathrm{CHN}), 3.93\left(1 \mathrm{H}, \mathrm{d}, \mathrm{J} 15.2, \mathrm{CH}_{2} \mathrm{Ph}\right), 3.76,(1 \mathrm{H}, \mathrm{d}, \mathrm{J} 2.8, \mathrm{OH})$, $3.16-3.08\left(4 \mathrm{H}, \mathrm{m}, \mathrm{CH}_{2} \mathrm{x} 2\right), 2.93\left(3 \mathrm{H}, \mathrm{s}, \mathrm{NCH}_{3}\right), 2.90(3 \mathrm{H}, \mathrm{s}$, $\left.\mathrm{NCH}_{3}\right), 2.89\left(3 \mathrm{H}, \mathrm{S}, \mathrm{NCH}_{3}\right), 2.84\left(3 \mathrm{H}, \mathrm{s}, \mathrm{NCH}_{3}\right) ; \delta_{\mathrm{C}}(100 \mathrm{MHz}$, $\left.\mathrm{CDCl}_{3}\right) \quad 158.7,138.0,136.4,128.6,128.4,128.1,128.0$, $127.7,127.6,87.9,82.1,75.2,74.6,72.9,68.0,64.7$, $64.3,53.6,52.6,52.3,52.0,51.6,46.8 ; \mathrm{m} / \mathrm{z}$ (ESI) $\left(\mathrm{M}+\mathrm{MeCN}+\mathrm{NH}_{4}^{+}, \quad 100 \%\right) ;$ HRMS for $\mathrm{C}_{27} \mathrm{H}_{38} \mathrm{~N}_{3} \mathrm{O}_{8} \mathrm{Os}$ requires 724.2274, found 724.2278 .

\section{Bn Diol (19)}

To a solution of osmate ester (17) (192 $\mathrm{mg}, 0.26 \mathrm{mmol}$ ) in DMF (7.5 mL) under an atmosphere of nitrogen was added benzyl bromide $(47.0 \mu \mathrm{L}, 0.40 \mathrm{mmol})$ and tetra $n$-butyl 
ammonium iodide $(10 \mathrm{mg})$. To this was added sodium hydride (32 mg, $1.33 \mathrm{mmol}$, pre-washed with petrol) in one portion and the resulting mixture was stirred at RT for $4 \mathrm{~h}$. The reaction was quenched with $\mathrm{H}_{2} \mathrm{O}(0.25 \mathrm{~mL})$, diluted with $\mathrm{MeOH}$ (50 $\mathrm{mL}$ ) and concentrated $\mathrm{HCl}$ (15 drops) was added and the solution stirred at RT for a further $3 \mathrm{~h}$. The reaction was diluted with saturated $\mathrm{NaHCO}_{3}(25 \mathrm{~mL})$ and $\mathrm{H}_{2} \mathrm{O}(50 \mathrm{~mL})$ then extracted with EtOAC $(30 \mathrm{~mL} \times 4)$. The combined organics were washed with brine (10 mL), dried over $\mathrm{MgSO}_{4}$ and concentrated onto slica. The crude material was purified by flash column chromatography, eluting with petrol:EtOAC (1:1 then 1:3) to furnish the title compound as a colourless oil (100.6 mg, $81 \%)$.

$[\alpha]_{\mathrm{D}}^{20}-48.9\left(\mathrm{C}=2.31, \mathrm{CHCl}_{3}\right) ; \boldsymbol{v}_{(\max )}$ (thin film) 3467, 1746

$\mathrm{Cm}^{-1} ; \delta_{\mathrm{H}}\left(400 \mathrm{MHz}, \mathrm{CD}_{3} \mathrm{CN}\right) 7.40-7.23(15 \mathrm{H}, \mathrm{m}, \mathrm{Ar}), 4.66-4.55$

$\left(4 \mathrm{H}, \mathrm{m}, \mathrm{CH}_{2} \mathrm{Ph}\right), 4.52(1 \mathrm{H}, \mathrm{app} . \mathrm{t}, \mathrm{J} 14.4, \mathrm{CHOC}), 4.35-4.32$ $\left(1 \mathrm{H}, \mathrm{m}, \mathrm{CH}_{2} \mathrm{Ph}\right), 4.11(1 \mathrm{H}, \mathrm{m}, \mathrm{C} \underline{\mathrm{HOH}}) 4.05-4.01\left(1 \mathrm{H}, \mathrm{m}, \mathrm{CH}_{2} \mathrm{Ph}\right)$, 4.02-3.99 (1H, m, CHN)，3.93-3.89 (1H, m, $\mathrm{m}$, CHOBn $\mathrm{x} 2), 3.56(1 \mathrm{H}, \mathrm{m}, \mathrm{OH}), 3.30(1 \mathrm{H}, \mathrm{m}, \mathrm{OH}) ; \delta_{\mathrm{C}}(100$ $\left.\mathrm{MHz}, \mathrm{CD}_{3} \mathrm{CN}\right) 159.2,138.6,138.4,137.6,129.0,128.8,128.7$, $128.4,128.3,128.2,128.2,128.1,127.8,78.1,76.8,74.8$, $72.4,72.2,70.8,68.9,55.4,46.8 ; \mathrm{m} / z \quad\left(E S I, \quad \mathrm{NH}_{3}\right)$ $\left(\mathrm{M}+\mathrm{MeCN}+\mathrm{NH}_{4}^{+}\right.$, $\left.100 \%\right) ;$ HRMS for $\mathrm{C}_{28} \mathrm{H}_{30} \mathrm{NO}_{4}$ requires 476.2073, found 476.2066 .

\section{(-) -Aminocyclitol (2)}

To a stirred solution of oxazolidinone (20) (50.5 mg, 0.10 mmol) in THF $(2.5 \mathrm{~mL})$ at $-78^{\circ} \mathrm{C}$ under an inert atmosphere of argon was distilled an excess of $\mathrm{NH}_{3}$. Strips of freshly cut Li metal (10 mg) were added and the solution turned deep blue over $10 \mathrm{~min}$. The reaction was stirred at $-78^{\circ} \mathrm{C}$ for a further $1.5 \mathrm{~h}$ before being carefully quenched with $\mathrm{H}_{2} \mathrm{O}$ (1.25 $\mathrm{mL}$ ). The reaction was allowed to warm to $\mathrm{RT}$, with the $\mathrm{NH}_{3}$ 
evaporating off, and then heated to $90^{\circ} \mathrm{C}$. After $1.5 \mathrm{~h}$, TLC analysis indicated consumption of the oxazolidinone and the reaction was cooled to $\mathrm{RT}$. The solution was neutralised and applied directly to a SCX IST column, eluting with MeOH then $\mathrm{MeOH}: 1 \mathrm{M} \mathrm{NH}_{4} \mathrm{OH}$ solution $(19: 1)$ to furnish the title compound as an amorphous solid (14.5 mg, 76\%).

$[\alpha]_{\mathrm{D}}^{20}-27.2\left(\mathrm{C}=0.45, \mathrm{H}_{2} \mathrm{O}\right) \quad\left[\right.$ lit. $[\alpha]_{\mathrm{D}}^{22}-33\left(\mathrm{C}=1.97, \mathrm{H}_{2} \mathrm{O}\right)^{1}$, $\left.[\alpha]_{\mathrm{D}}^{21}-28.9\left(\mathrm{C}=0.85, \mathrm{H}_{2} \mathrm{O}\right)^{4}\right] ; V_{(\max )}$ (neat) $3387 \mathrm{~cm}^{-1} ; \delta_{\mathrm{H}}(400$ $\mathrm{MHz}, \mathrm{D}_{2} \mathrm{O}$, with acetone as internal standard at $\left.\delta 2.08 \mathrm{ppm}\right)$ $5.07(1 \mathrm{H}, \mathrm{s}), 4.84(1 \mathrm{H}, \mathrm{s}), 4.17(1 \mathrm{H}, \mathrm{dd}, \mathrm{J} 7.6,4.8), 4.07$ $(1 \mathrm{H}, \mathrm{dd}, \mathrm{J} 4.4,4.4), 4.01(1 \mathrm{H}, \mathrm{dd}, J 10.0,4.0), 3.73(1 \mathrm{H}$, $\mathrm{dd}, J 10.0,3.6), 3.60(1 \mathrm{H}, \mathrm{dd}, J 8.0,3.2), 3.25(1 \mathrm{H}, t, J$ $3.2) ; \delta_{\mathrm{C}}\left(125 \mathrm{MHz}, \mathrm{CD}_{3} \mathrm{OD}\right)$ 95.5, 79.3, 78.6, 71.9, 71.7, 70.1, 56.1. This data was consistent with the literature values. 


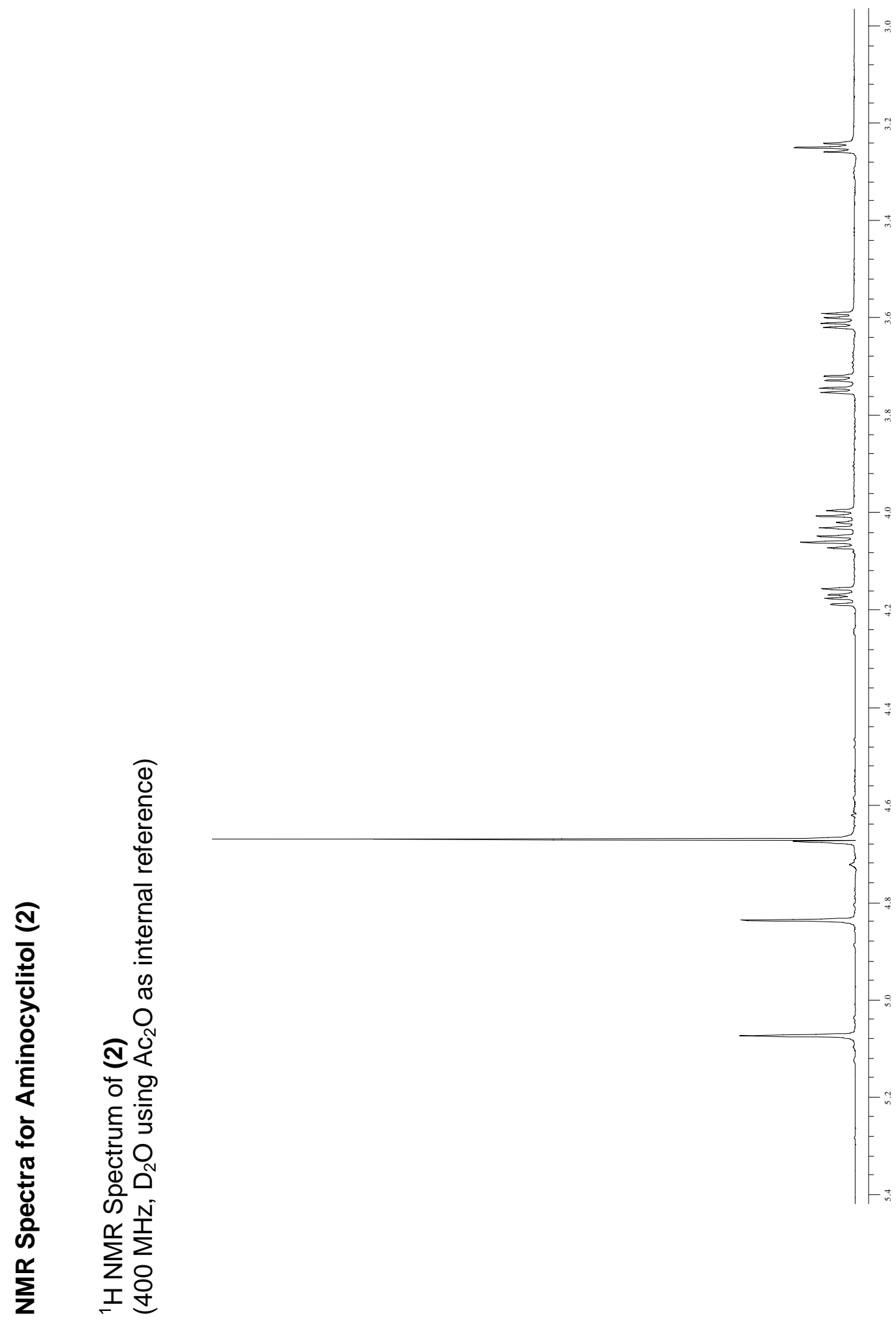




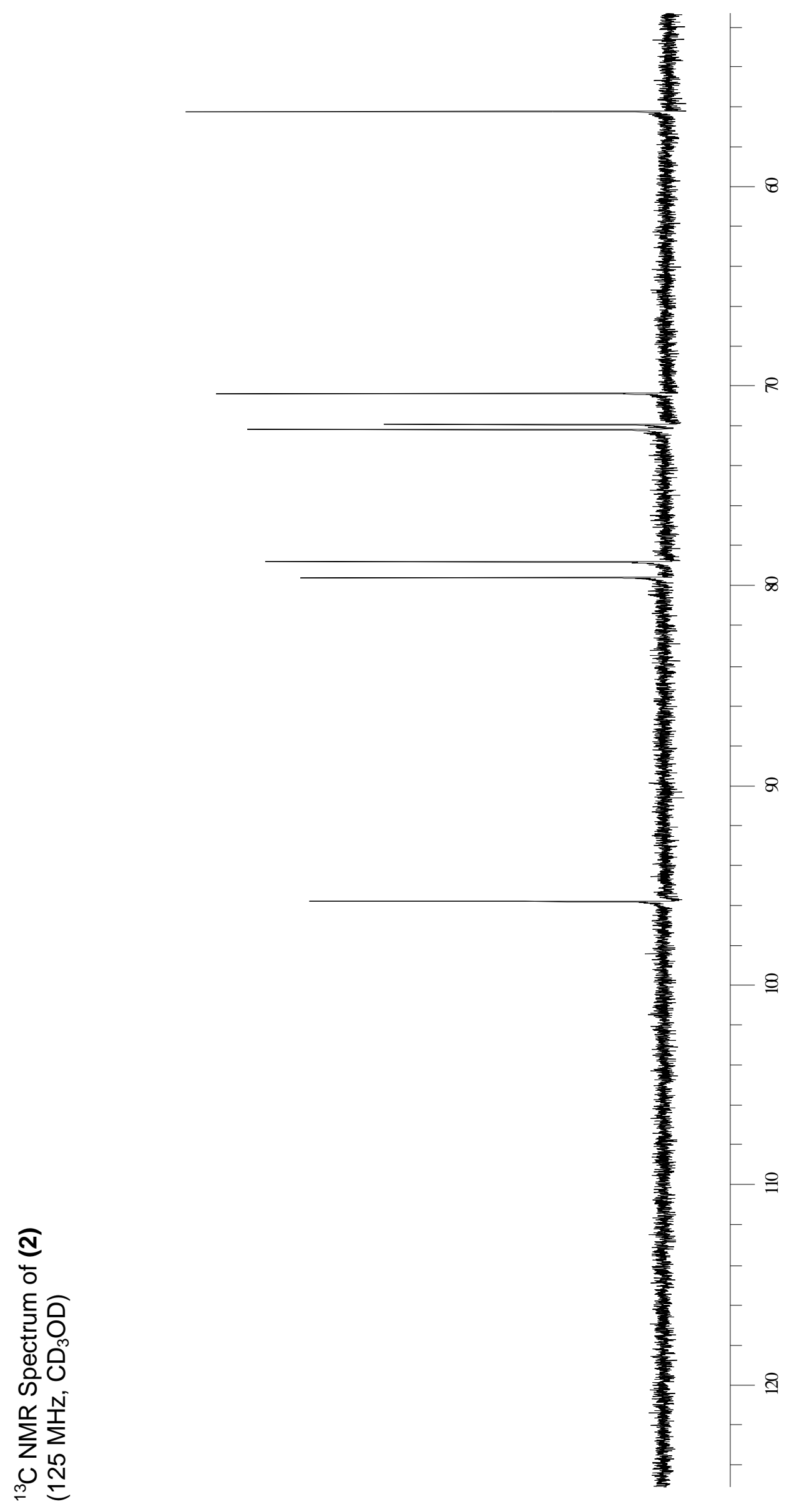




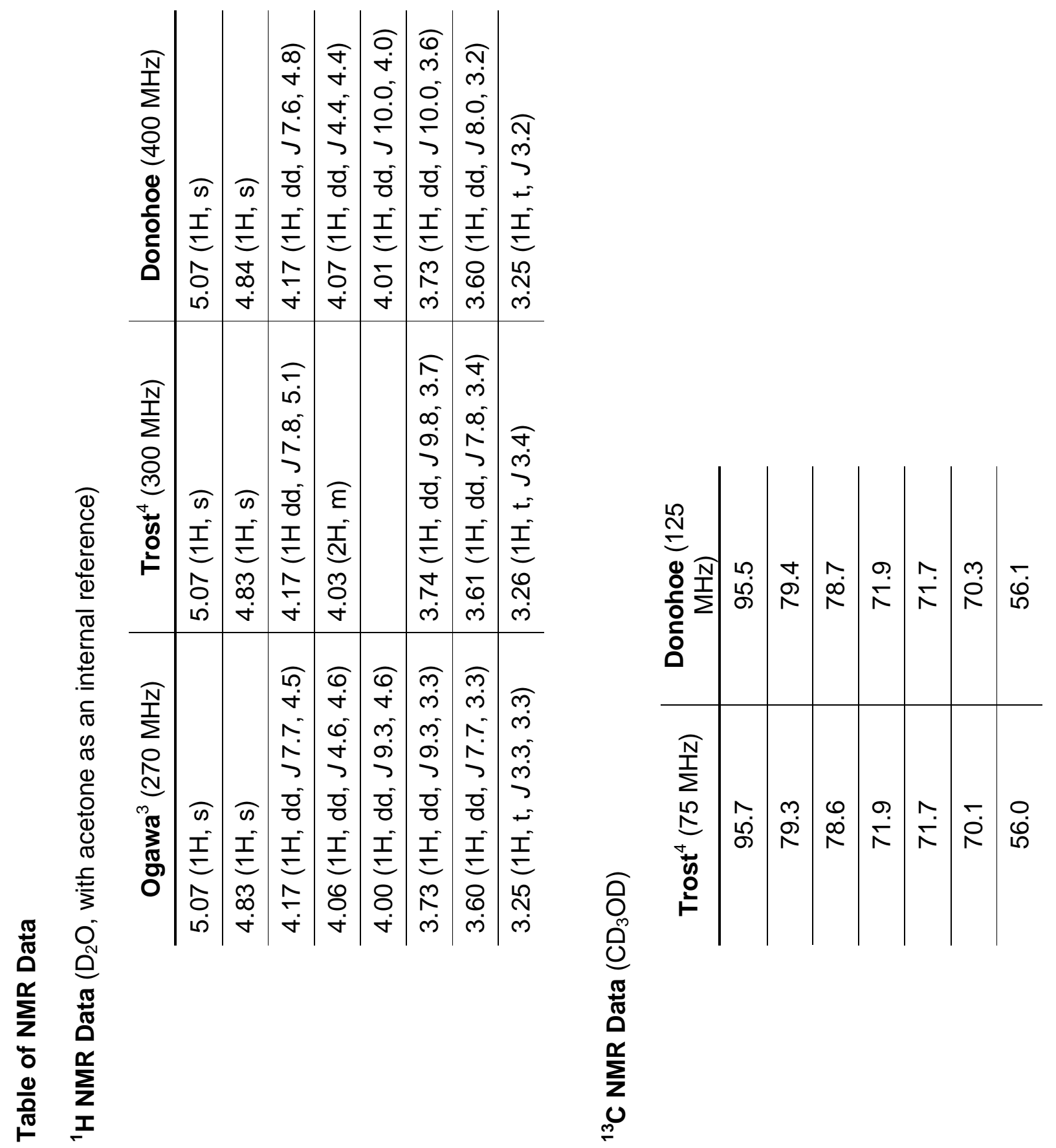




\section{References}

1. R. C. Pittenberger, R. N. Wolfe, M. M. Hohen, P. N. Marks, W. A. Daily, M. McGuire, Antibiot. Chemother. 1953， 3， 1268; R. L. Mann, R. M. Gale, F. R. VanAbeele, Antibiot. Chemother. 1953, 3, 1279; Y. Sumiki, G. Nakamura, M. Kawasaki, S. Yamashita,K. Anazi, K. Isono, Y. Serizawa, Y. Tomiyama, S. Suzuki, J. Antibiot. 1955, 8, 170; K. Isono, S. Yamashita, Y. Tomiyama, S. Suzuki, J. Antibiot. 1957, 10, 21; Y. Wakisaka, K. Koizumi, Y. Nishimoto, M. Kobayashi, N. Tsuji, J. Antibiot. 1980, 33, 695.

2. K. Kakinuma, Y. Sakagami, Agric. Biol. Chem., 1978, 42,279 .

3. N. Chida, M. Ohtsuka, K. Nakazawa, S. Ogawa, J. Org. Chem., 1991, 56, 2976.

4. B. M. Trost, J. Dudash, Jr., E. J. Hembre, Chem. Eur. J., 2001, 7, 1619. 